\title{
Auf dem Weg zur sexuellen Selbstbestimmung: Das Engagement der Bundeszentrale für gesundheitliche Aufklärung (BZgA) für Menschen mit Beeinträchtigungen
}

\section{On the Way to Sexual Self-Determination: The Commitment of the Federal Centre for Health Education (BZgA) for People with Disabilities}

\section{(ㄷ) (1) $\odot \odot$}

\author{
Autor:innen \\ Institut \\ Bundeszentrale für gesundheitliche Aufklärung (BZgA), Köln \\ Schlüsselwörter \\ Beeinträchtigungen; Behinderungen; Partizipation; \\ Sexualaufklärung; sexuelle und reproduktive Gesundheit \\ Keywords \\ disabilities; impairments; participation; sex education; \\ sexual and reproductive health \\ Bibliografie \\ Z Sexualforsch 2021; 34: 163-167 \\ DOI 10.1055/a-1551-7132 \\ ISSN 0932-8114 \\ (c) 2021. The Author(s). \\ This is an open access article published by Thieme under the terms \\ of the Creative Commons Attribution-NonDerivative-NonCommer- \\ cial-License, permitting copying and reproduction so long as the \\ original work is given appropriate credit. Contents may not be used \\ for commercial purposes, or adapted, remixed, transformed or built \\ upon. (https://creativecommons.org/licenses/by-nc-nd/4.0/) \\ Georg Thieme Verlag KG, Rüdigerstraße 14, \\ 70469 Stuttgart, Germany
}

Sara Scharmanski, Stefanie Paschke, Mirjam Tomse und Laura Brockschmidt

\author{
Korrespondenzadresse \\ Dr. rer. nat. Sara Scharmanski \\ Bundeszentrale für gesundheitliche Aufklärung (BZgA) \\ Maarweg 149-161 \\ 50825 Köln \\ Deutschland \\ sara.scharmanski@bzga.de
}

\section{ZUSAMMENFASSUNG}

Jeder Mensch hat das Recht auf den Zugang zu evidenzbasierten Informationen zur Sexualaufklärung, Verhütung und Familienplanung. Im Sinne eines ganzheitlichen Verständnisses von sexueller und reproduktiver Gesundheit fördert die Bundeszentrale für gesundheitliche Aufklärung (BZgA) mit ihren Maßnahmen das Recht auf eine selbstbestimmte Sexualität von Menschen mit Beeinträchtigungen. Der vorliegende Praxisbeitrag stellt diese Aktivitäten und Materialien zusammenfassend dar.

\section{ABSTRACT}

Everyone has the right to access evidence-based information on sexuality education, contraception and family planning. In the spirit of a holistic understanding of sexual and reproductive health, the Federal Centre for Health Education (BZgA) promotes the right to self-determined sexuality of people with impairments through its measures. This practice-oriented article summarizes these activities and materials.
Das menschliche Bedürfnis nach Sexualität wurde als universelles Menschenrecht in der UN-Behindertenrechtskonvention (UN-BRK) explizit formuliert. Demnach haben Menschen mit Beeinträchtigungen das Recht, über alle Fragen zu Ehe, Partnerschaft, Familie und Elternschaft selbst zu entscheiden. Die Aktivitäten der Bundeszentrale für gesundheitliche Aufklärung $(\mathrm{BZgA})$ in diesem Feld sind Bestandteil des 2011 gestartete Nationalen Aktionsplans (NAP) der Bundesregierung zur Umsetzung der UN-BRK. Ziel ist der Abbau von Barrieren in allen Lebensbereichen von Menschen mit Beeinträchtigungen.

Vor diesem Hintergrund und dem des gesetzlichen Auftrags aus dem Schwangerschaftskonfliktgesetz (§1 Abs. 1 SchKG) werden in der BZgA seither vielfältige Medien und Maßnahmen entwickelt und umgesetzt, um Menschen mit Beeinträchtigungen in ihrer selbstbestimmten Sexualität zu unterstützen und eine zielgruppengerechte, ganzheitliche Sexualaufklärung zu fördern. Ziel dieses En- 
gagements ist es, Menschen mit Beeinträchtigungen dabei zu unterstützen, sich im Themenfeld Sexualität eigenständig zu informieren und selbstbestimmte Entscheidungen treffen zu können.

Damit dies gelingt, wurde ein holistischer Ansatz zur Umsetzung des Themas „Sexualität und Beeinträchtigungen“ gewählt, der von den allgemeinen Grundsätzen der Gesundheitsförderung Empowerment, Partizipation und Kompetenzausbau geprägt ist (Abel et al. 2018; Brandes und Stark 2016; Wright 2016). Dieser Ansatz basiert auf mehreren Säulen:

- dem Public Health Action Cycle als Grundlage bei der Qualitätsentwicklung und -sicherung aller Interventionen und Maßnahmen (Rosenbrock und Hartung 2015),

- der Entwicklung von Konzepten zur Sexualaufklärung, in denen die Voraussetzungen zur Befähigung eines eigen- und partnerverantwortlichen und gesundheitsförderlichen Umgangs mit Sexualität dargelegt werden,

- der Entwicklung von barrierearmen Angeboten für verschiedene Zielgruppen (wie z. B. Fachkräfte, Kinder, Jugendliche und Erwachsene),

- der Förderung von Forschungs- und Praxisprojekten zur Erweiterung der sexuellen Selbstbestimmung und Sexualaufklärung,

- der partizipativen und evidenzbasierten Entwicklung einzelner Maßnahmen,

- der Bearbeitung der Themenkomplexe auf nationaler und internationaler Ebene.

Der vorliegende Praxisbeitrag stellt das Engagement der BZgA zur Förderung der sexuellen und reproduktiven Gesundheit von Menschen mit Beeinträchtigungen zusammenfassend dar: Zunächst wird die konzeptuelle Grundlage, das Rahmenkonzept zur Sexualaufklärung der BZgA, vorgestellt. Anschließend geht es um partizipative Forschung, die Bedeutung von Digitalisierung, Qualitätssicherung und der internationalen Arbeit in diesem Themenbereich.

\section{Das Rahmenkonzept zur Sexualaufklärung der BZgA}

Das Rahmenkonzept zur Sexualaufklärung (BZgA 2016) wurde unter Beteiligung aller Bundesländer erarbeitet. Darauf basierend wurden spezifische Konzepte für besondere Zielgruppen entwickelt. Im Konzept zur Sexualaufklärung von Menschen mit Beeinträchtigungen (BZgA 2015) wird zu Rahmenbedingungen und Begriffsbestimmungen von Menschen mit Beeinträchtigungen Bezug genommen. Das Thema „Behinderung“ wird hier als Lebenserfahrung verstanden und deren Auswirkungen auf Sexualität, Partnerschaft und Elternschaft werden konkretisiert. Das Konzept zur Sexualaufklärung von Menschen mit Beeinträchtigungen ist als Version in Brailleschrift und Großdruck sowie in Leichter Sprache erhältlich. Durch die (barrierefreien) Versionen des Rahmenkonzeptes wird der gemeinsame Austausch von Menschen mit und ohne Beeinträchtigungen über Themen der Sexualaufklärung gefördert. Zudem stellen sie die Grundlage für ein aktives und partizipatives Zusammenarbeiten in Forschungs- und Praxisprojekten dar.

\section{Partizipative Forschung}

Ein wichtiger Baustein der Aktivitäten der BZgA sind die wissenschaftlichen Analysen und Ergebnisse zur sexuellen und reproduktiven Gesundheit von Menschen mit Beeinträchtigungen. Sie bilden das Fundament, um qualitätsgesicherte und evidenzbasierte Konzepte und Aufklärungsmaßnahmen zur Verfügung zu stellen. Dabei ist es wichtig, die verschiedenen Zielgruppen, ihre Lebenswelten und die Bedarfe durch die jeweiligen Beeinträchtigungsformen adäquat zu berücksichtigen. Dies erfordert nicht nur die Beachtung der unterschiedlichen Kommunikationsmittel oder Wohnkontexte, sondern die aktive Partizipation der jeweiligen Zielgruppen an wissenschaftlicher Forschung. So können die Lebens- und Erlebenswirklichkeiten von Menschen mit Beeinträchtigungen im Forschungsprozess besonders reichhaltig berücksichtigt werden.

Diesen Prämissen ist die BZgA unter anderem bei der Förderung der folgenden drei barrierefrei und partizipativ angelegten Studien der Universität Leipzig gefolgt:

- Jugendsexualität und Behinderung. Eine Studie zur besonderen Situation von behinderten Jugendlichen in Sachsen (Wienholz et al. 2013),

- Familienplanung bei jungen Erwachsenen mit Behinderungen in Sachsen (Wienholz et al. 2017),

- Teilhabechancen an sexueller Bildung Jugendlicher mit kognitiven Einschränkungen in Sachsen (Wienholz 2017).

Als Expertinnen und Experten in eigener Sache wurden Jugendliche und junge Erwachsene mit kognitiven Beeinträchtigungen in die einzelnen Forschungsschritte eingebunden. Im Ergebnis konnten die Erlebenswirklichkeiten dieser Zielgruppen in Bezug auf Sexualaufklärung, Verhütung sowie Gewalterfahrungen in die Studien einfließen (Paschke und Tomse 2017). Derartige empirische Studienergebnisse bilden dann wiederum die Grundlage für Praxismaßnahmen der BZgA.

\section{Digitalisierung zur Förderung der sexuellen Selbstbestimmung: Das „ReWiKs“-Projekt}

Menschen mit Beeinträchtigungen sind in ihrer sexuellen Selbstbestimmung und dem Zugang zu evidenzbasierten Informationen zu Themen der Sexualaufklärung nach wie vor mit vielfältigen Barrieren konfrontiert. Vor allem Menschen, die in Wohneinrichtungen leben, müssen institutionell-strukturelle Hindernisse (z. B. Mangel an Privatsphäre, Kultur und Haltung der Einrichtung, knappe personelle Ressourcen), aber auch individuell-persönliche Barrieren vonseiten der Einrichtungen, Fachkräfte und Leitungspersonen (z. B. Unsicherheit, Ängste gegenüber dem Thema „Sexualität von Menschen mit Beeinträchtigungen“) überwinden (Charitou et al. 2021; Greenwood und Wilkinson 2013; Ortland et al. 2016).

Zudem belegen Studien, dass vor allem Frauen mit Beeinträchtigungen und Bewohnerinnen und Bewohner in Wohneinrichtungen einem erhöhten Risiko für sexualisierte Gewalt ausgesetzt sind (Schröttle et al. 2013) und häufiger aversive sexuelle Erfahrungen machen (Holdsworth et al. 2018).

Um den Unterstützungsbedarfen sowohl der Menschen mit Beeinträchtigungen als auch der Mitarbeitenden in Wohneinrichtungen gerecht zu werden, wird seit 2014 das von der BZgA geförderte Forschungsprojekt „ReWiKs (Reflexion - Wissen - Können)“ zur 
Stärkung der sexuellen Selbstbestimmung bei erwachsenen Menschen mit Beeinträchtigungen in Wohneinrichtungen gefördert (Ortland et al. 2016). In einem intensiven Wissenschafts-Praxis-Dialog und unter konsequenter Einbeziehung von Menschen mit Beeinträchtigungen wurde ein umfangreiches Medienpaket erstellt. Diese Materialen dienen dazu,

- Mitarbeitende zur Reflexion des intentionellen Umgangs mit Sexualität anzuregen und ihnen Fachwissen und Handlungsempfehlungen bereitzustellen sowie

- Bewohner:innen zu unterstützen, ihr Recht auf eine selbstbestimmte Sexualität einzufordern und Entwicklungsprozesse in ihren Wohneinrichtungen anzustoßen bzw. an diesen zu partizipieren.

Im Zuge der Entwicklung der ReWiKs-Materialien wurde geprüft, inwieweit durch die Verwendung von digitalen Materialien und onlinebasierten Zugängen die Partizipation von Menschen mit Beeinträchtigungen am Organisationsentwicklungsprozess gesteigert werden könnte. Verstärkt wurde diese Notwendigkeit durch die COVID-19-Pandemie: Kontaktbeschränkende Maßnahmen machten ein Mehr an Digitalisierung unabdingbar. Zudem bietet das Internet für Menschen mit Beeinträchtigungen einen hohen Anreiz und eine Chance, selbstbestimmt und autonom zu interagieren, zu kommunizieren und an gesellschaftlichen Prozessen teilhaben zu können (Manzoor und Vimarlund 2018). Trotz dieser möglichen Chancen zeigen aktuelle Studienergebnisse aber auch, dass die Mediennutzung von Menschen mit Beeinträchtigungen stark eingeschränkt ist (Bosse et al. 2019). Gerade in stationären Wohneinrichtungen haben Menschen mit Beeinträchtigungen deutlich seltener Zugang zu digitalen Medien und sind auf die Unterstützung von Betreuer:innen angewiesen (Adrian et al. 2017).

Diese Zugangs- und Nutzungsbarrieren für Menschen mit Beeinträchtigungen zeigten sich auch im ReWiKs-Projekt sehr deutlich. Im Rahmen einer explorativen Online-Befragung von N=68 Mitarbeitenden in Wohneinrichtungen (Kemmerling und Bössing 2020) wurden Hürden sowohl in Bezug auf die Beeinträchtigungen der Bewohner:innen als auch auf die technische Infrastruktur der Einrichtungen (z. B. Verfügbarkeit von WLAN) sichtbar. Und auch auf Ebene der Mitarbeitenden bestehen Barrieren: Diese Zielgruppe ist in Bezug auf die eigene Mediennutzungs- und Medienvermittlungskompetenz sowie die Einstellung gegenüber digitalen Medien sehr heterogen. Nicht zuletzt - und das zeigte das ReWiKs-Projekt auch sehr deutlich - bestimmen die Kultur und die Haltung innerhalb der Einrichtung sowie die verfügbaren personellen Ressourcen den Zugang der Bewohner:innen zu digitalen Medien.

Auf Basis dieser Ergebnisse wurden die Konzeptionen der digitalisierten ReWiKs-Materialien angepasst. Als erster Schritt hin zu einer komplett digitalen Nutzungsmöglichkeit wurden die Entwicklung von barrierearmen digitalen Dokumenten und eine barrierearme Online-Präsenz identifiziert und umgesetzt ${ }^{1}$. Dies setzte voraus, dass sowohl die inhaltliche Ebene (z. B. Leichte Sprache, Ein-

1 Während der aktuellen Erprobungsphase stehen alle Materialien barrierearm in einem geschlossenen Online-Portal für Teilnehmende der ReWiKs-Fortbildung zur Verfügung. Weitere Informationen unter https:// www.reha.hu-berlin.de/de/lehrgebiete/kbp/forschung/rewiks-1/ fortbildungen. bindung von Bildern und Grafiken) als auch die technische Ebene (z. B. Nutzbarkeit mit Screenreadern und assistiven Technologien, wie beispielsweise Eyegaze-Systeme) in den Blick genommen werden musste. Als Wegweiser bei der barrierearmen Digitalisierung des ReWiKs-Medienpakets dienten dabei die Vorgaben der „Web Content Accessibility Guideline“ (WCAG) (Caldwell et al. 2008).

Der Digitalisierungsprozess der ReWiKs-Materialen ist - wie der gesamte ReWiKs-Forschungsprozess - iterativ angelegt; d. h., es finden kontinuierliche Evaluationen und Anpassungen sowohl der digitalen Materialien als auch der Online-Präsenz statt. Die zentrale Frage, die bei der Entwicklung und der Evaluation handlungsleitend ist, lautet: Wie schaffen wir es, unter den bestehenden strukturell-organisatorischen Rahmenbedingungen die Erreichbarkeit von Menschen mit Beeinträchtigungen durch Digitalisierung zu verbessern?

\section{Qualitätssicherung durch Partizipation: Das Projekt „Herzfroh 2.0“}

Die Digitalisierung von Informationen zum Abbau von Barrieren ist ein zentraler Bestandteil des Projekts „Herzfroh 2.0“. Es sollen digitale und analoge Informationsangebote für Jugendliche und junge Erwachsene mit kognitiven Beeinträchtigungen entstehen. In Kooperation mit den Departements Soziale Arbeit und Informatik der Hochschule Luzern werden die Themenhefte des Manuals „Herzfroh“2 zur Sexualaufklärung aus der Schweiz überarbeitet und weiterentwickelt. Begleitend zu den Themenheften und interaktiven Tools werden eine pädagogische Handreichung und haptisches Begleitmaterial für die schulische und außerschulische Arbeit entwickelt. Die Informationen sollen so verständlich und barrierearm aufbereitet und zur Verfügung gestellt werden, dass sie von Menschen mit kognitiven Beeinträchtigungen eigenständig genutzt und gleichzeitig mit passenden Medien und Methoden von Fachkräften gut vermittelt werden können.

So ergeben sich zwei zentrale Zielgruppen für die spätere Nutzung: Sowohl Jugendliche und junge Erwachsene mit kognitiven Beeinträchtigungen als auch Fachkräfte werden bereits bei der Überarbeitung und Neuentwicklung der Medien von „Herzfroh 2.0“ eingebunden. Darüber hinaus wird die Medienentwicklung über die gesamte Projektlaufzeit von den Bildungsfachkräften des Instituts für Inklusive Bildung in Kiel (https://inklusive-bildung.org/ de/ueber-uns) begleitet. Sie testen die Medien und Formate und beraten das Projektteam bei der Umsetzung. So können notwendige Anpassungen bereits im Entwicklungsprozess erkannt und umgesetzt werden.

Die Partizipation ist dabei Teil der Qualitätssicherung. Zentral hierbei ist, die Zielgruppen zu verschiedenen Zeitpunkten und auf verschiedenen Ebenen einzubeziehen: Bereits in der ersten Projektphase wurden relevante Personengruppen in den Entwicklungsprozess involviert. Die bestehenden Materialien wurden geprüft und

2 „Herzfroh - Fragen und Antworten rund um Körper, Sex und Liebe“ ist ein sexualpädagogisches Manual, bestehend aus 17 Themenheften zu verschiedenen Schwerpunkten. Entwickelt wurde das Manual zwischen 2007 und 2011 von der Fachstelle Behinderung und Sexualität (fabs) in der Schweiz. Nach der Auflösung der Fachstelle im Jahr 2011 übernahm die Hochschule Luzern - Soziale Arbeit die Weiterführung und Weiterentwicklung der Materialien. 
bewertet, um den Bedarf an Überarbeitung und Neuentwicklung von Teilbereichen möglichst breit zu ermitteln. Unter Einbezug des international und multiprofessionell besetzten Fachbeirates wurden Themenerweiterungen sowie Einsatzfelder der Medien diskutiert. In Fokusgruppen mit Fachkräften in Deutschland und in der Schweiz wurden die Themenhefte in Bezug auf Darstellungen, Sprache und Aufbau diskutiert. In Fokusgruppen mit Jugendlichen und jungen Erwachsenen mit kognitiven Beeinträchtigungen wurden die Themenhefte in Wohngruppen besprochen und testweise im Förderschulunterricht eingesetzt. So konnten wertvolle Rückmeldungen zur Verständlichkeit der Medien gesammelt werden. Alle Rückmeldungen wurden ausgewertet und fließen in den Entwicklungsprozess ein. Aktuell werden die ersten analogen und digitalen Medien auf Grundlage der Ergebnisse umgesetzt.

In der zweiten Projektphase werden die entwickelten Medien von den Bildungsfachkräften und in Fokusgruppen erneut getestet, um die Überarbeitungen und Neuentwicklungen zu bewerten. Noch vor der Veröffentlichung der Medien ist eine weitere Überarbeitungsschleife vorgesehen, in der die Rückmeldungen umgesetzt werden können.

Die bisherigen Erfahrungen mit dem partizipativen und iterativen Entwicklungsprozess im Projekt „Herzfroh 2.0“ zeigen, dass die Einbindung der verschiedenen Zielgruppen sehr zeit- und personalintensiv ist. Ein solcher Entwicklungsprozess sichert jedoch die Bereitstellung von Medien, die genau auf die Bedarfe der jeweiligen Zielgruppe ausgerichtet sind. So kann eine hohe Akzeptanz und Nutzbarkeit der Medien gewährleistet werden. Partizipation von Menschen mit Beeinträchtigungen und Fachkräften ist vor diesem Hintergrund für eine qualitätsgesicherte Entwicklung von Informationen zur Sexualaufklärung unerlässlich.

\section{Das internationale Engagement der BZgA}

Als WHO-Kollaborationszentrum für sexuelle und reproduktive Gesundheit (WHO CC) 3 fördert die BZgA seit 2003 die Implementierung von ganzheitlicher Sexualaufklärung in der europäischen Region der Weltgesundheitsorganisation (WHO) (Ketting et al. 2018).

Das WHO CC in der BZgA entwickelt in enger Kooperation mit dem europäischen WHO-Regionalbüro, einer internationalen Gruppe aus Expert:innen und weiteren Partner:innen, Maßnahmen für die Sexualaufklärung in Europa und tritt für die Forschung und Praxis in diesem Bereich ein. Seit seiner Ernennung hat das WHO CC zahlreiche internationale Publikationen vorgelegt. ${ }^{4}$ Besonders hervorzuheben ist die Entwicklung der „Standards für Sexualaufklärung in Europa“, die im selben Maße für Menschen mit und ohne Beeinträchtigungen Gültigkeit haben (WHO Regional Office for Europe und BZgA 2010).

Im Rahmen der aktuellen Schwerpunktsetzung hat das WHORegionalbüro für Europa den Zugang zu Sexualaufklärung für Kinder und Jugendliche mit Beeinträchtigungen auf die politische Agenda gesetzt (WHO Regional Office for Europe 2016). Vor diesem Hin-

3 Das WHO-Regionalbüro Europa ernennt die sogenannten WHO-Kollaborationszentren. Diese Zentren führen dann in einem bestimmten Zeitraum Programme zu einzelnen gesundheitsbezogenen Schwerpunktthemen durch.

4 Siehe www.bzga-whocc.de/publikationen. tergrund legt das WHO CC in der BZgA seit 2019 einen besonderen Fokus auf diese Zielgruppe. Den Ausgangspunkt für das Engagement des WHO CC stellt ein von der BZgA beauftragtes systematisches Literaturreview dar. Erste Ergebnisse machen deutlich, dass Kinder und Jugendliche mit Beeinträchtigungen auch im europäischen Raum oftmals keine Sexualaufklärung erhalten oder Angebote nicht an ihre Bedürfnisse angepasst sind (Michielsen und Brockschmidt 2021). Zudem bildet das systemische Literaturreview die Grundlage für weitere Publikationen im Bereich Sexualaufklärung für Kinder und Jugendliche mit Beeinträchtigungen in der WHO CC Region, welche aktuell in Planung sind. Auch auf nationalen und internationalen Fachkonferenzen, die von der BZgA mitorganisiert werden, wird das Thema aufgegriffen. So kamen auf der UNESCOTagung „Switched On“ zur sexuellen Bildung im digitalen Raum im Jahr 2020 unter anderem Influencer:innen mit Behinderungen zu Wort, die auf Sozialen Medien wie YouTube sexualbezogene PeerEducation betreiben (Döring 2020).

Das Recht auf ganzheitliche Sexualaufklärung für alle Menschen ist in internationalen Übereinkommen verankert (z. B. WHO Regional Office for Europe 2016). Während das Thema „Sexualität von Menschen mit Beeinträchtigungen“ damit politisch auf internationaler Ebene an Relevanz gewinnt, ist die praktische Umsetzung in den meisten Ländern noch nicht weit vorangeschritten (UNFPA 2018). Das WHO CC hat es sich zur Aufgabe gemacht, die Bedarfe und Rechte international verstärkt sichtbar zu machen und die Umsetzung von Sexualaufklärung für Menschen mit Beeinträchtigungen in nationalen Richtlinien und Programmen zu fördern.

\section{Fazit und Ausblick}

Auf Basis der UN-BRK, dem NAP der Bundesregierung und dem SchKG setzt sich die BZgA seit 2012 für die sexuelle Selbstbestimmung und die sexuelle und reproduktive Gesundheit von Menschen mit Beeinträchtigungen ein. Das nationale und internationale Engagement beinhaltet dabei sowohl die Entwicklung und Abstimmung von Rahmenkonzepten als auch die Umsetzung von Forschungsvorhaben und die Entwicklung von barrierefreien Materialien, Angeboten und Qualifizierungsmaßnahmen für Fachkräfte. Gemäß dem partizipativen Ansatz werden bei allen Maßnahmen Personen mit Beeinträchtigungen eng in die Entwicklungs- und Evaluationsprozesse eingebunden. So können sie ihre Lebenswirklichkeiten als Fachleute in eigener Sache einbringen.

Noch immer sind Jugendliche und Erwachsene mit Beeinträchtigungen bezüglich ihrer Rechte auf sexuelle Selbstbestimmung und sexuelle und reproduktive Gesundheit mit zahlreichen Hemmnissen und Hürden konfrontiert. Die BZgA wird sich auch in Zukunft gemeinsam mit Vertreter:innen der Länder und Verbänden sowie im Rahmen von nationalen und internationalen Kooperationen dafür einsetzen, allen Menschen einen gleichwertigen Zugang zu Informationen der Sexualaufklärung, Verhütung und Familienplanung zu ermöglichen.

\section{Interessenkonflikt}

Alle Autorinnen sind bei der BZgA beschäftigt und teilweise Leiterinnen der beschriebenen Projekte. 
Literatur

Abel T, Jordan S, Sommerhalder K, Bruhin E. Health Literacy/Gesundheitskompetenz. In: [BZgA] Bundeszentrale für gesundheitliche Aufklärung, Hrsg. Leitbegriffe der Gesundheitsförderung und Prävention. Köln: BZgA 2018; 582-587

Adrian S, Hölig S, Hasebrink U, Bosse I, Haage A. Mediennutzung von Menschen mit Beeinträchtigungen. Medienbezogene Handlungen, Barrieren und Erwartungen einer heterogenen Zielgruppe. Media Perspektiven 2017; Heft 3: 145-156

Bosse I, Zaynel N, Lampert C. Mediennutzung und Vermittlung von Medienkompetenz in der Behindertenhilfe in Bremen. merz 2019; 63: 24-31

Brandes S, Stark W. Empowerment/Befähigung. In: [BZgA] Bundeszentrale für gesundheitliche Aufklärung, Hrsg. Leitbegriffe der Gesundheitsförderung und Prävention. Köln: BZgA 2016; 62-65

[BZgA] Bundeszentrale für gesundheitliche Aufklärung. Sexualaufklärung von Menschen mit Beeinträchtigungen. Köln: BZgA 2015

[BZgA] Bundeszentrale für gesundheitliche Aufklärung. Rahmenkonzept zur Sexualaufklärung der Bundeszentrale für gesundheitliche Aufklärung in Abstimmung mit den Bundesländern. Köln: BZgA 2016

Caldwell B, Cooper M, Reid LG, Vanderheiden G. Web Content Accessibility Guidelines (WCAG) 2.0. Cambridge, MA: W3C 2008[Als Online-Dokument: https:/www.w3.org/TR/2008/REC-WCAG20-20081211/]

Charitou M, Quayle E, Sutherland A. Supporting Adults with Intellectual Disabilities with Relationships and Sex: A Systematic Review and Thematic Synthesis of Qualitative Research with Staff. Sex Disabil 2021; 39: 113-146

Döring N. „Switched On“: UNESCO-Konferenz 2020 zur sexuellen Bildung im digitalen Raum. Z Sexualforsch 2020; 33: 178-180

Greenwood NW, Wilkinson J. Sexual and Reproductive Health Care for Women with Intellectual Disabilities: A Primary Care Perspective. Int J Family Med 2013; 2013: 642472

Holdsworth E, Trifonova V, Tanton C, Kuper H, Datta J, Macdowall W, Mercer $\mathrm{CH}$. Sexual Behaviours and Sexual Health Outcomes among Young Adults with Limiting Disabilities: Findings from third British National Survey of Sexual Attitudes and Lifestyles (Natsal-3). BMJ Open 2018, 8: e019219

Kemmerling M, Bössing C. Auswertung des Kurzfragebogens Digitalisierung im Projekt ReWiKs. Köln 2020; unveröffentlichtes Manuskript

Ketting E, Brockschmidt L, Renner I, Luyckfasseel L, Ivanova O. Sexuality Education in Europe and Central Asia: Recent Developments and Current Status. In: Benavides-Torres R, Onofre-Rodrígez DJ, Márquez-Vega MA, Barbosa-Martìnez RC, Hrsg. Sex Education. Global Perspectives, Effective Programs and Socio-Cultural Challenges. New York, NY: Nova Science Publishers, Inc. 2018; 75-120

Manzoor M, Vimarlund V. Digital Technologies for Social Inclusion of Individuals with Disabilities. Health Technol 2018; 8: 377-390

Michielsen K, Brockschmidt L. Barriers to Sexuality Education for Children and Young People with Disabilities in the WHO European Region: A Scoping Review. Sex Educ 2021; doi:10.1080/14681811.2020.18511 81

Ortland B, Jennessen S, Romisch K, Kusber-Merkens D, Reichert L, Arlabosse A. Das Modellprojekt ReWiKs. Aktuelle Forschungsergebnisse zu Teilhabechancen Erwachsener mit Behinderung im Bereich der sexuellen Selbstbestimmung. Bundesgesundheitsblatt Gesundheitsforschung Gesundheitsschutz 2016, 59: 1085-1092

Paschke S, Tomse M. Sexualaufklärung für Menschen mit Beeinträchtigungen. Die Aktivitäten der Bundeszentrale für gesundheitliche Aufklärung (BZgA) im Themenfeld. BZgA Forum Sexualaufklärung und Familienplanung 2017; Heft 1: 3-5

Rosenbrock R, Hartung S. Public Health Action Cycle/Gesundheitspolitischer Aktionszyklus. In: [BZgA] Bundeszentrale für gesundheitliche Auf- klärung, Hrsg. Leitbegriffe der Gesundheitsförderung und Prävention. Köln: BZgA 2015; 833-835

Schröttle M, Glammeier S, Honberg C, Kavemann B, Puhe H, Zinsmeister J. Lebenssituation und Belastungen von Frauen mit Beeinträchtigungen und Behinderungen in Deutschland. Ergebnisse der quantitativen Befragung. Endbericht. Berlin: BMFSFJ 2013

[UNFPA] United Nations Populations Fund. Young Persons with Disabilities: Global Study on Ending Gender-Based Violence, and Realising Sexual and Reproductive Health and Rights. New York, NY: UNFPA 2018

[WHO] World Health Organization Regional Office for Europe. Action Plan for Sexual and Reproductive Health: Towards Achieving the 2030 Agenda for Sustainable Development in Europe - Leaving No One Behind. Kopenhagen: WHO Regional Office for Europe 2016

[WHO] World Health Organization Regional Office for Europe, [BZgA] Bundeszentrale für gesundheitliche Aufklärung. Standards for Sexuality Education in Europe. A Framework for Policy Makers, Educational and Health Authorities and Specialists. Köln: BZgA 2010

Wienholz S. Sexuelle Bildung aus Perspektive von Jugendlichen und jungen Erwachsenen mit kognitiven Beeinträchtigungen und deren Hauptbezugspersonen. In: BZgA Forum Sexualaufklärung und Familienplanung 2017; Heft 1: 39-42

Wienholz S, Seidel A, Michel M. Junge Erwachsene mit Beeinträchtigungen. Sexualität · Verhütung · Soziale Beziehungen. Ausgewählte Ergebnisse einer Befragung in Sachsen. Onlineversion. Köln: BZgA 2017 [Als Online-Dokument: https://www.forschung.sexualaufklaerung.de/ fileadmin/fileadmin-forschung/pdf/Kurzfassung_Junge_Erwachsene_ mit_Beeintraechtigungen_Sachsen_Onlineversion.pdf]

Wienholz S, Seidel A, Michel M, Müller M. Jugendsexualität und Behinderung. Ergebnisse einer Befragung an Förderschulen in Sachsen. Köln: BZgA 2013

Wright M. Partizipation: Mitentscheidung der Bürgerinnen und Bürger. In: [BZgA] Bundeszentrale für gesundheitliche Aufklärung, Hrsg. Leitbegriffe der Gesundheitsförderung und Prävention. Köln: BZgA 2016; 707-711 